\title{
Área de figuras planas com objetos de aprendizagem no Geogebra
}

\section{RESUMO}

Agostinho laqhan Ryokiti Homa laqchan@hotmail.com

Universidade Luterana do Brasil, Canoas, Rio Grande do Sul, Brasil

Claudia Lisete Oliveira Groenwald claudiag1959@yahoo.com.br

Universidade Luterana do Brasil, Canoas, Rio Grande do Sul, Brasil

\begin{abstract}
Este artigo apresenta uma sequência didática em formato digital, de acordo com o padrão SCORM, implementada na plataforma de ensino ILIAS, com a temática área de figuras planas, utilizando objetos de aprendizagem, construídos no software Geogebra. O objetivo foi elaborar uma sequência didática, com objetos de aprendizagem interativos, que levassem os estudantes do Ensino Fundamental a construírem os conceitos de polígonos e as fórmulas de áreas das figuras planas através da observação e manipulação desses objetos. Toda a sequência encontra-se disponível no endereço: http://matematica.ulbra.br/ilias.
\end{abstract}

PALAVRAS-CHAVE: Geogebra. Sequência Didática Eletrônica. Objetos de Aprendizagem. Área de Figuras Planas. 


\section{INTRODUÇÃO}

Kampff et al. (2004) afirmam que em uma sociedade de bases tecnológicas, com mudanças contínuas, não é mais possível desprezar o potencial pedagógico que as Tecnologias de Informação e Comunicação (TIC) apresentam quando incorporadas à educação. Assim, o computador é um instrumento pertinente no processo de ensino e aprendizagem, cabendo à escola utilizá-lo de forma coerente com uma proposta pedagógica atual e comprometida com uma aprendizagem significativa.

Este artigo apresenta uma sequência didática eletrônica, organizada em conformidade com o padrão SCORM 2004 e implementada na plataforma de ensino ILIAS, com a temática Polígonos e Área de figuras planas, utilizando objetos de aprendizagem, construídos no software Geogebra.

O objetivo foi elaborar uma sequência didática eletrônica, com objetos de aprendizagem interativos, que levassem os estudantes do Ensino Fundamental a construírem o conceito de polígonos e as fórmulas de áreas das figuras planas através da observação e manipulação desses objetos.

\section{SEQUÊNCIA DIDÁTICA}

Entende-se por Sequência Didática a organização de um conteúdo qualquer, a partir da articulação entre os conceitos e procedimentos a serem desenvolvidos, com atividades didáticas planejadas para esse fim, com foco na aprendizagem. Segundo Zabala (1998, p. 18), sequências didáticas são "[...] um conjunto de atividades ordenadas, estruturadas e articuladas para a realização de certos objetivos educacionais, que tem um princípio e um fim conhecido, tanto pelos professores como pelos alunos". Dolz e Schneuwly (2004) consideram que sequências didáticas são organizadas pelo professor com o objetivo de alcançar a aprendizagem de seus alunos, e envolvem atividades de aprendizagem e avaliação.

Segundo Groenwald et al. (2009, p.2):

A vantagem do uso de uma sequência didática em uma plataforma de ensino é a possibilidade da utilização de diferentes recursos, com padrão superior de qualidade, como vídeos-exemplo, textos com exemplos em movimento, ou seja, um conteúdo visual com maior qualidade de visualização.

Com a intenção de prover acesso a um aprendizado de alta qualidade, levando em consideração as necessidades individuais, a capacidade de ser entregue a qualquer hora e em qualquer lugar a um custo efetivo menor do então existente, em 1977 o Departamento de Defesa dos Estados Unidos (DoD) e o Escritório de Política de Ciência e Tecnologia da Casa Branca (OSTP) lançaram o ADL (Advanced Distributed Learning Initiative). Esse objetivo é alcançado com a definição do SCORM (Sharable Content Object Reference Model), que define o conteúdo reutilizável como um objeto educacional dentro de uma estrutura técnica para aprendizagem baseada em computador e na Web (ADLNET, 2004).

O SCORM é o resultado da especificação e padronização baseadas no trabalho de outras entidades normativas, podendo ser identificado como a união das melhores práticas e padrões existentes em e-learning (electronic learning) anteriores ao seu surgimento (ADLNET, 2004); padrões que continuam sendo 
aprimorados pelos seus criadores, os quais colaboram com o ADL Initiative. O SCORM 2004 3rd Edition é a edição mais atual e sua adesão por parte dos desenvolvedores de sistemas está aumentando em relação à versão anterior, de 2002, que já está bem difundida (ADLNET, 2004).

Uma sequência de apresentação de conteúdos em conformidade com o padrão SCORM garante a portabilidade da sequência, ou seja, garante o mesmo comportamento de apresentação em qualquer LMS (Learning Management System) que também esteja em conformidade com o padrão (ADLNET, 2004). Atualmente existem diversos LMS, de uso livre e com tecnologias proprietárias, em conformidade com o SCORM, dentre as de uso livre o Moodle e o ILIAS são destaque. Neste trabalho optou-se pela plataforma ILIAS, pois a mesma tem um gerenciamento mais simples que o Moodle com uma interface visualmente menos carregada e possibilidade de customização das telas dos usuários.

O uso de atividades interativas, para a sequência didática, levou à busca por um DGS (Dynamic Geometry System - Sistema de Geometria Dinâmica) para o desenvolvimento das atividades em formato eletrônico. Deste modo, para atender as recomendações do padrão SCORM, as atividades foram desenvolvidas utilizando o software Geogebra, pois o mesmo permite que as atividades sejam exportadas para um formato WEB.

O Geogebra é um software open source, sob o GNU (General Public License) dispovível em www.geogebra.org, que agrega as funcionalidades de DGS e de Computer Algebric System (CAS) no plano, sendo então denominado como um Dynamic Mathematics Software (DMS) para Geometria, Álgebra e Cálculo (HOHENWARTER; PREINER, 2007).

Segundo Hohenwater e Fuchs (2004):

\begin{abstract}
Geogebra é um software de Geometria interativa que também fornece possibilidades algébricas como entrar diretamente com equações. Ele é direcionado aos estudante (10 a 18 anos) e professores do Ensino Médio. 0 software incentiva os estudantes a abordarem a matemática de maneira experimental (tradução nossa).
\end{abstract}

A sequência didática foi organizada de modo que, através de atividades interativas, o aluno identifique as relações entre as dimensões das figuras planas e a área das mesmas. Para tal objetivo, as atividades foram desenvolvidas como objetos de aprendizagem segundo o conceito de granularidade (ADLNET, 2004) e como objetos específicos de aplicação, desenvolvidos com base em um objetivo simples (HODGINS, 2002) de modo que cada atividade é independente entre si podendo ser reutilizado em outras sequências didáticas.

Os objetos de aprendizagem são partes componentes dessa organização que, segundo Willey (2000), são recursos digitais que podem ser reutilizados para o suporte ao desenvolvimento do processo de ensino e aprendizagem. Para o IEEE (Institute of Electrical and Electronics Engineers, 2000) os objetos de aprendizagem são qualquer entidade, digital ou não, que pode ser utilizada, reutilizada ou referenciada durante o processo de ensino e aprendizagem que utilize tecnologia.

As atividades são compostas de instruções para que os alunos entendam quais são as interações disponíveis e o que cada informação representa podendo o 
deve ter um objetivo de aprendizagem dentro de um determinado tempo para a sua execução.

Logo as atividades têm a característica de reutilização, com uma descrição mínima de seus objetivos, tipos de interações, existindo em si só, ou seja, não depende de outros objetos para atender o objetivo proposto.

Merrill (2000) afirma que objetos sem um design instrucional são somente objetos de conhecimento, ou seja, tem um caráter mais informativo, por isso a sequência didática segue uma abordagem construtivista, onde o estudante através das interações que realiza com os objetos de aprendizagem identifica as relações e generaliza os conceitos propostos.

\section{ORGANIZAÇÃO DA SEQUÊNCIA DIDÁTICA COM A TEMÁTICA POLÍGONOS E ÁREA DE FIGURAS PLANAS}

Na sequência desenvolvida os objetos estão encadeados didaticamente, levando o estudante a construção dos conceitos iniciais de polígono e de área de figuras planas.

Os conceitos abordados foram: linha poligonal, polígono (conceito, classificação), triângulo (conceito, classificação, elementos, área); quadriláteros (conceito, classificação, elementos, área), circunferência e círculo (conceito e área).

Os objetos de aprendizagem foram desenvolvidos em uma página HTML, organizado em dois campos, a esquerda o objeto do Geogebra e a direita são apresentados os conceitos e instruções de interação com o objeto.

A seguir apresenta-se a sequência desenvolvida.

\subsection{LINHA POLIGONAL E POLÍGONO}

O objetivo é que o estudante identifique linha poligonal, tipos de linhas poligonais, conceito de polígono. A figura 1 apresenta o objeto de aprendizagem e as manipulações possíveis com este objeto. 
Figura 1 - Objeto de Aprendizagem de Linha Poligonal

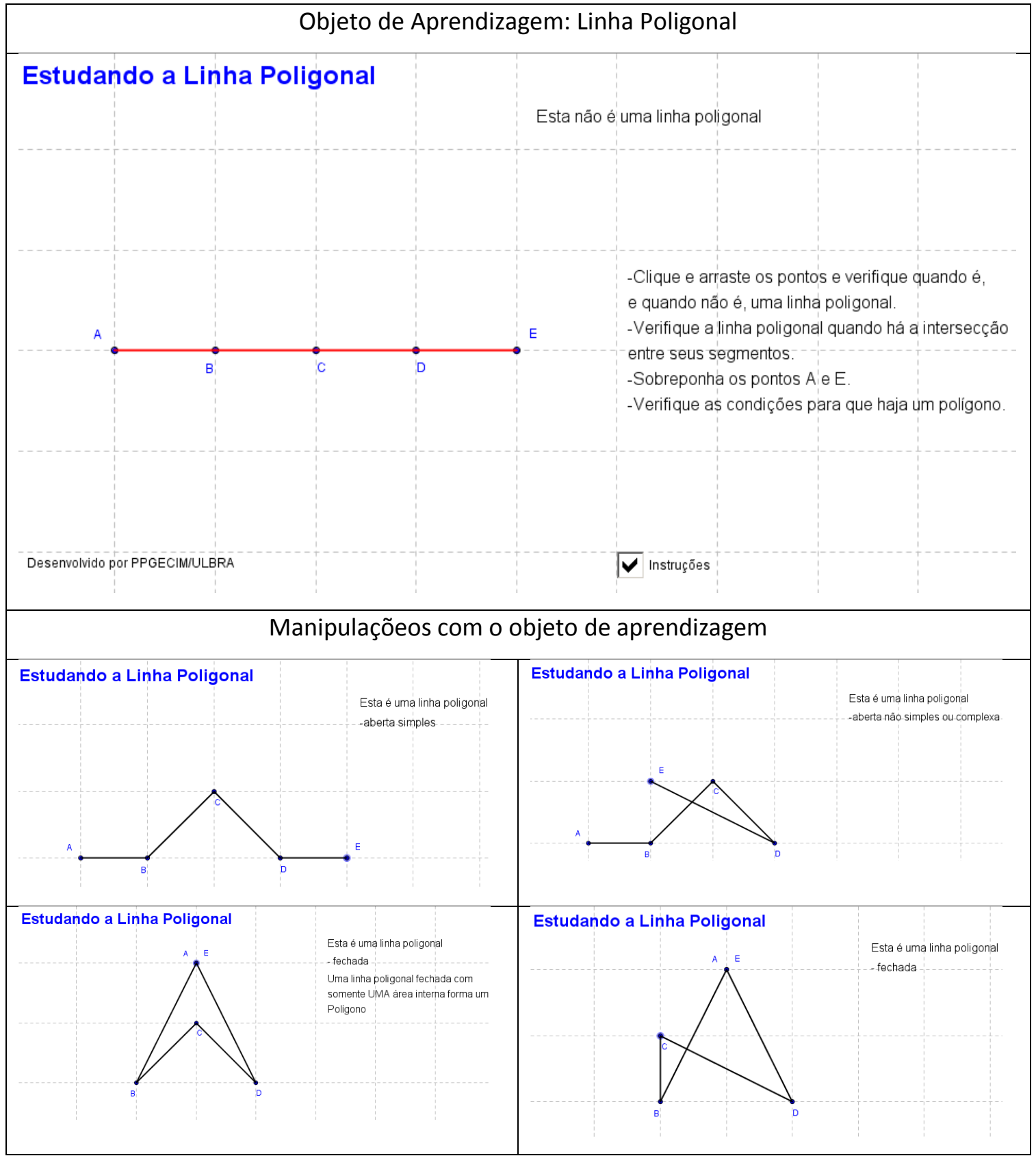

Fonte: Repositório de Objetos de Aprendizagem do PPGECIM.

As conclusões possíveis com as manipulações do objeto referido são:

- Linha poligonal é uma linha formada por um conjunto de segmentos de retas sucessivas e não colineares.

- Classificação das Linhas Poligonais: linha poligonal aberta simples; linha poligonal aberta complexa (quando há intersecção entre os segmentos); linha poligonal fechada;

- Polígono é uma figura plana limitada por uma linha poligonal fechada com uma região interna. 


\subsection{TIPOS DE POLÍGONOS}

O objetivo desta atividade é que o estudante identifique polígonos côncavos e convexos. A figura 2 apresenta o objeto de aprendizagem e as manipulações possíveis com este objeto.

Figura 2 - Objeto de Aprendizagem com tipos de Polígonos

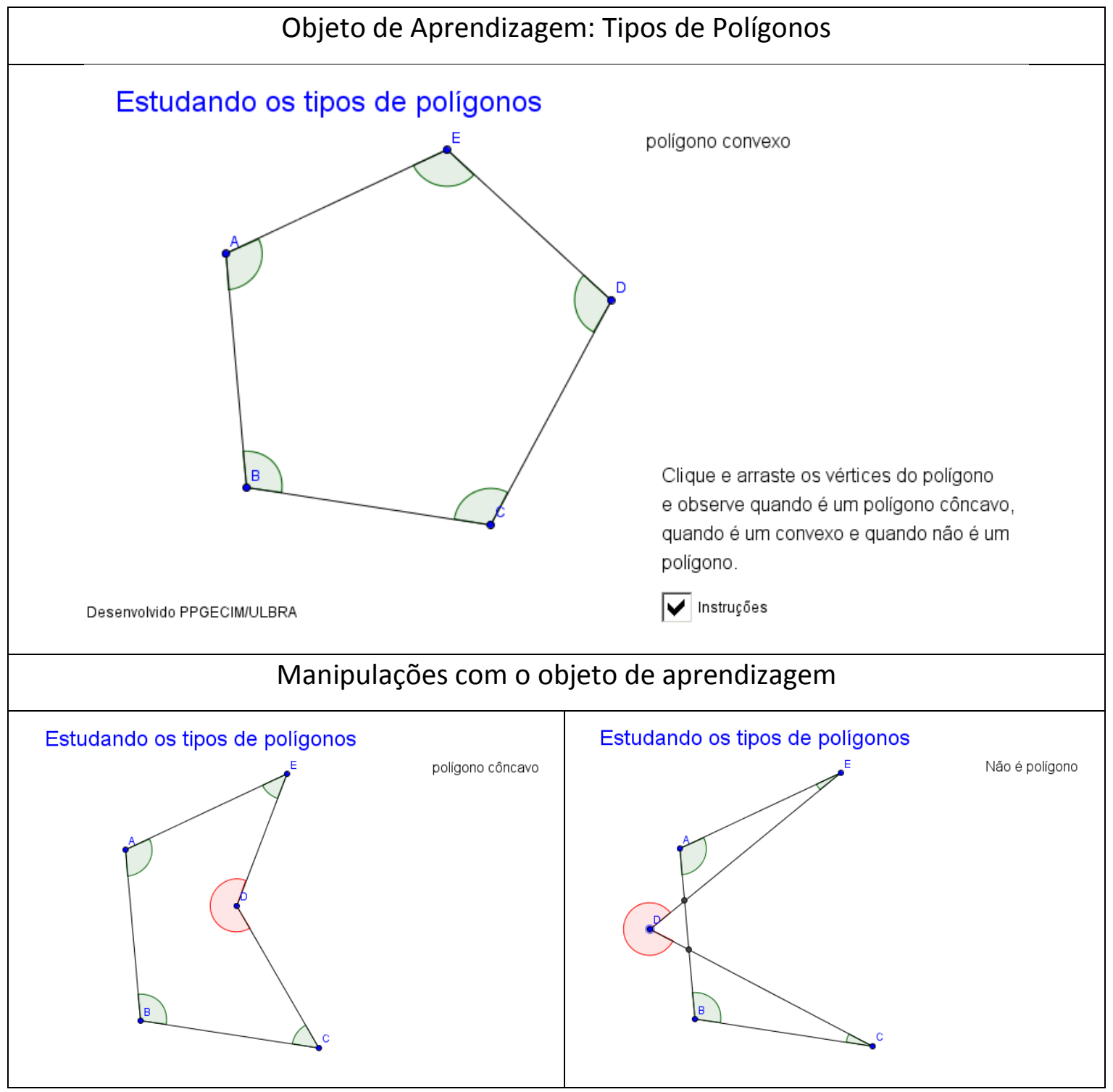

Fonte: Repositório de Objetos de Aprendizagem do PPGECIM.

As conclusões possíveis são:

- Os polígonos podem ser classificados em côncavos e convexos.

- Um polígono convexo possui todos os ângulos internos menores que $180^{\circ} \mathrm{e}$ um polígono côncavo possui pelo menos um dos ângulos internos maiores que 180 ㅇ. 
- Quando uma linha poligonal possui mais de uma região interna não é um polígono.

\subsection{CLASSIFICAÇÃO DOS POLÍGONOS}

O objetivo desta atividade é que o estudante, manipulando o cursor $n^{\circ}$ de lados, identifique a classificação dos polígonos regulares e irregulares de acordo com o número de lados e conheça a nomenclatura dada aos polígonos. A Figura 3 apresenta o objeto e as manipulações com o objeto desenvolvido. 
Figura 1 - Objeto com classificação de Polígonos

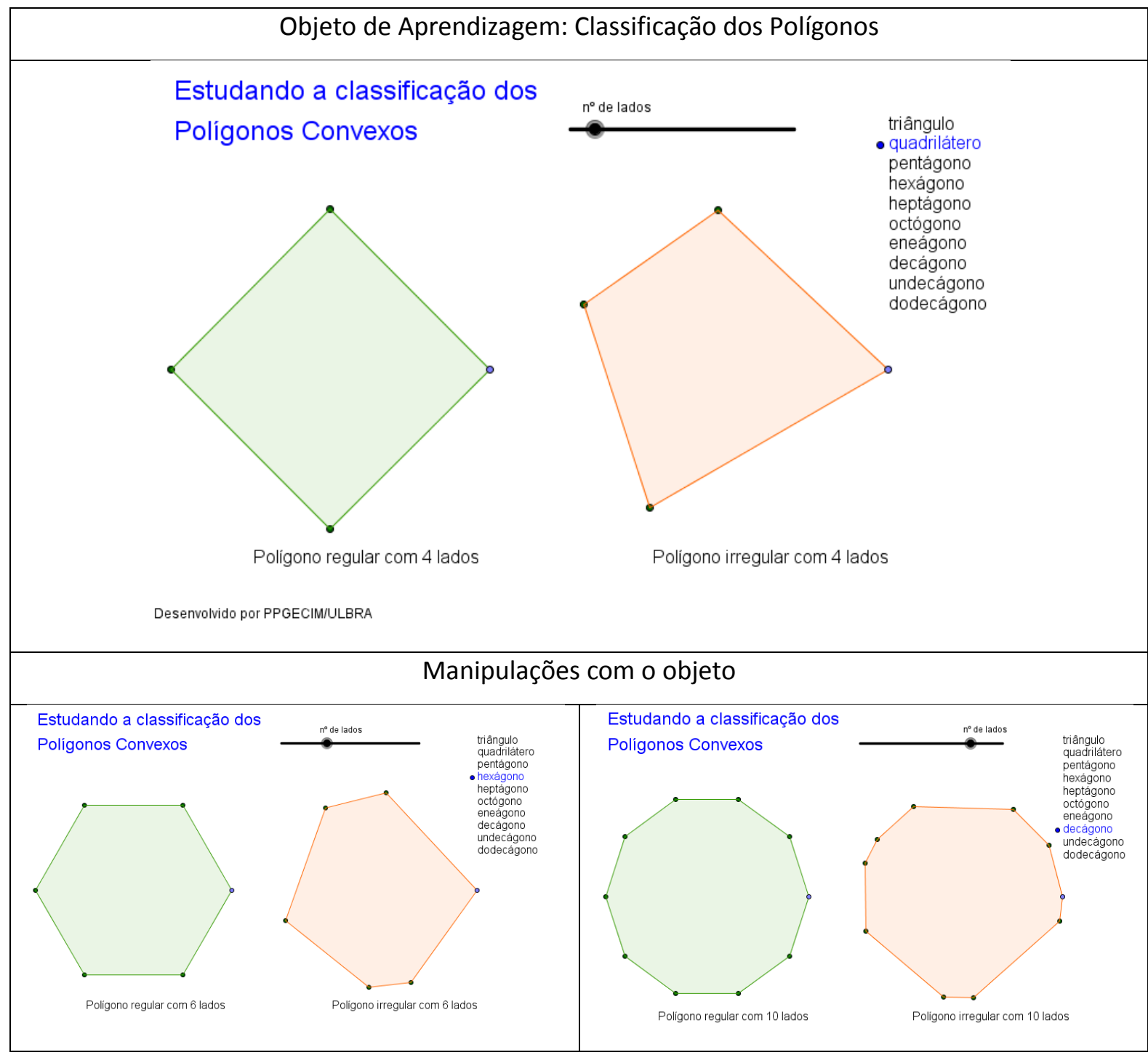

Fonte: Repositório de Objetos de Aprendizagem do PPGECIM.

\subsection{TRIÂNGULOS}

Essa atividade objetiva que o estudante identifique a classificação dos triângulos de acordo com os lados e os ângulos. A Figura 4 apresenta o objeto desenvolvido e as manipulações que podem ser realizadas. 
Figura 4 - Objeto de Aprendizagem com Triângulos

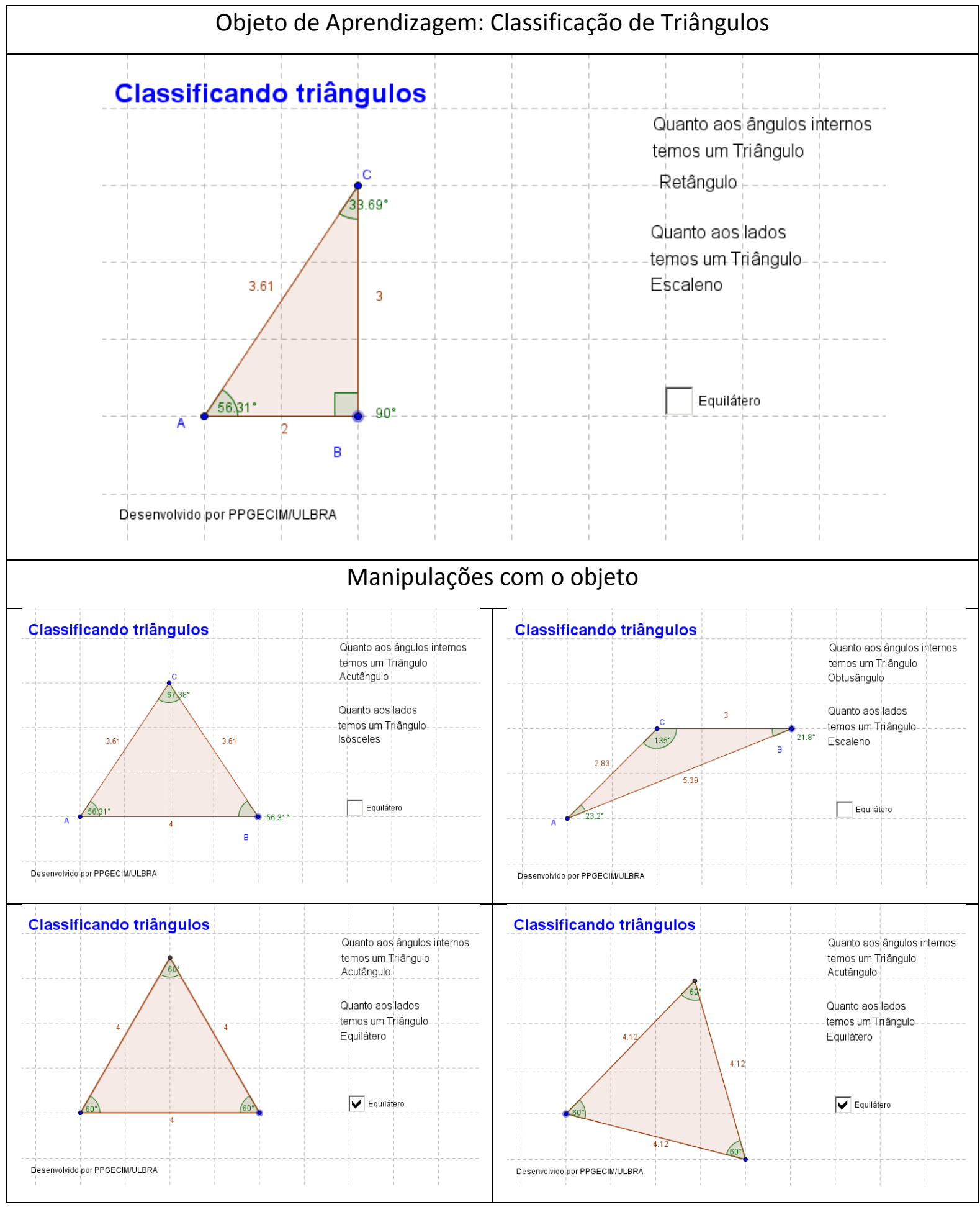

Fonte: Repositório de Objetos de Aprendizagem do PPGECIM. 
Neste objeto, o estudante consegue construir os triângulos escaleno e isósceles com facilidade, porém, como os vértices só podem ser posicionados sobre a malha, não se consegue desenhar o triângulo equilátero. Por isso optou-se pelo uso de um botão para o desenho do triângulo equilátero, no qual é possível a manipulação de dois vértices sobre a malha com o terceiro vértice vinculado a estes.

\subsection{QUADRILÁTEROS}

O objetivo é que o estudante através das interações com o objeto identifique a classificação dos quadriláteros convexos de acordo com suas propriedades. A Figura 5 apresenta o objeto de aprendizagem com classificação dos quadriláteros convexos.

Figura 5 - Objeto de aprendizagem com Quadriláteros Convexos

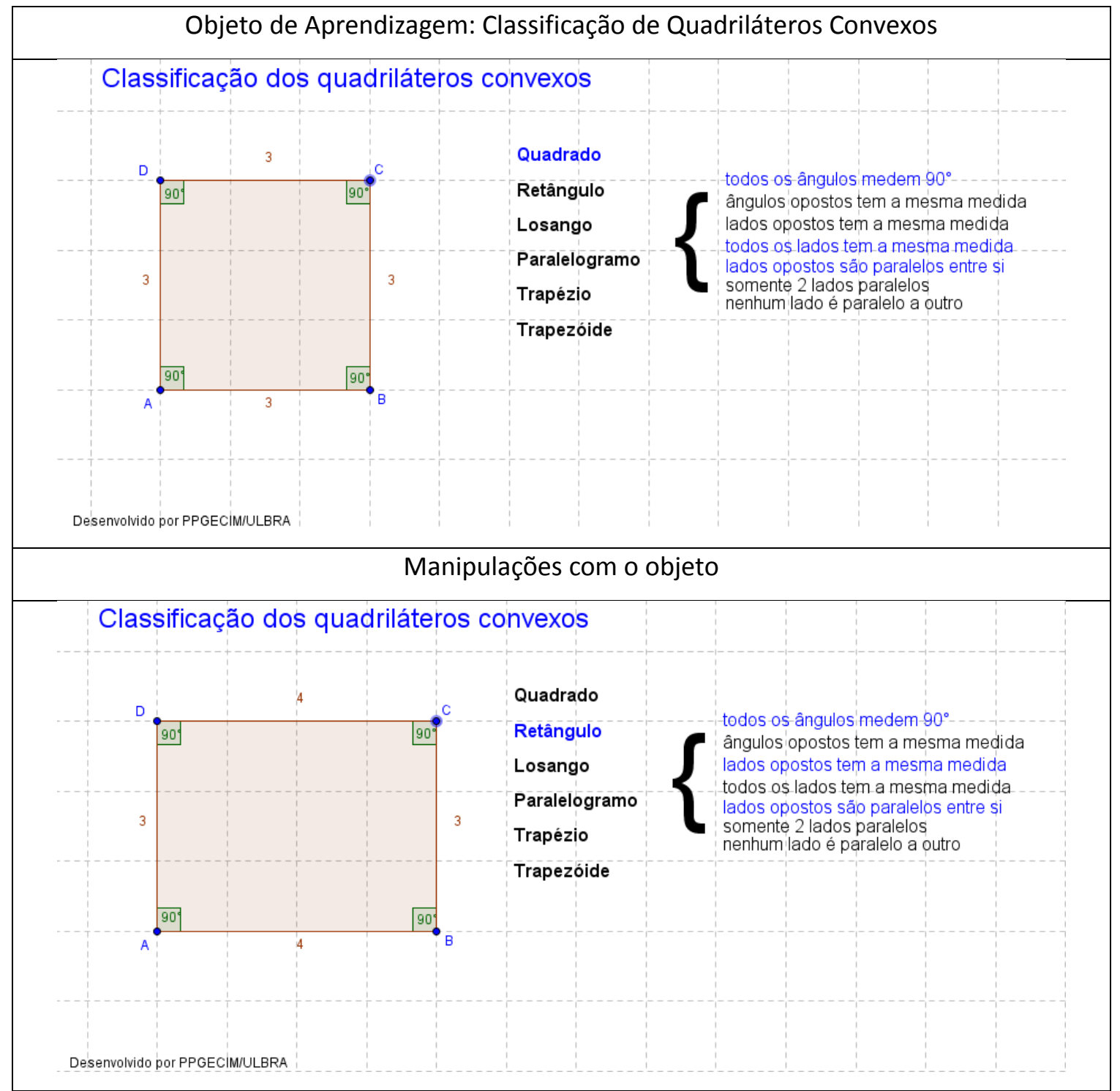




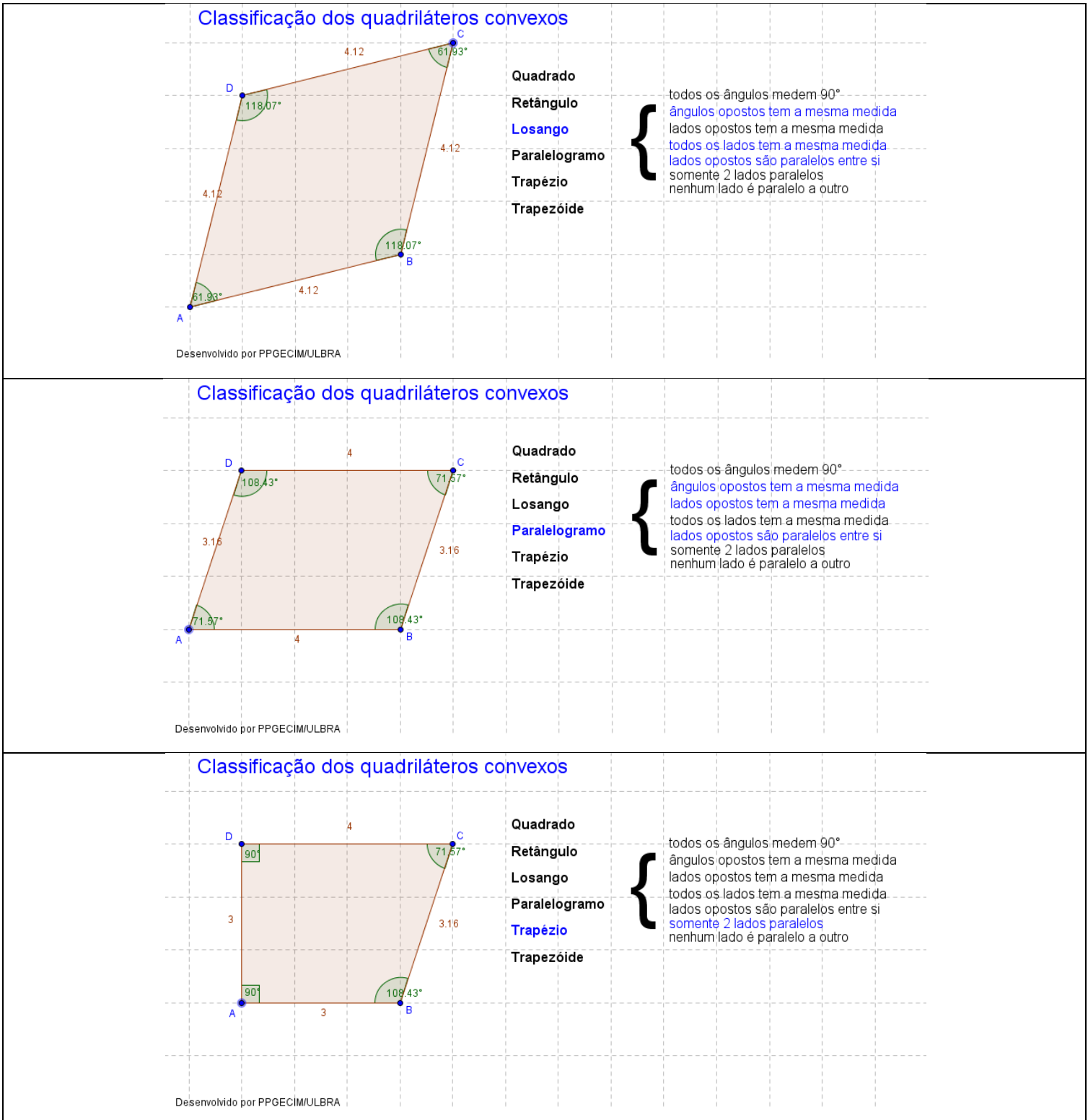

Classificação dos quadriláteros convexos

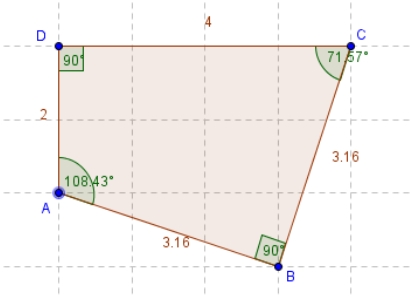

Quadrado

Retângulo todos os ângulos medem $90^{\circ}$

ângulos opostos tem a mesma medid

Losango

Paralelogramo

Trapézio

dos opostos tem a mesma medida

lados opostos săo paralelos entre si nenhum lado é paralelo a out

Trapezóide

Desenvolvido por PPGECIM/ULBRA

Fonte: Repositório de Objetos de Aprendizagem do PPGECIM. 
Optou-se por apresentar toda a classificação e propriedades dos quadriláteros para que o estudante identifique as propriedades existentes, em azul, no quadrilátero formado e visualize, simultaneamente, as propriedades não existentes no quadrilátero.

\section{6 Área de figuras planas}

Os objetos de aprendizagem a seguir visam que o estudante visualize o modelo matemático para área de figuras planas. Foram desenvolvidos na seguinte ordem: retângulos e quadrados, paralelogramos propriamente dito, trapézios, losangos, triângulos e círculos. A Figura 6 apresenta o objeto de aprendizagem para área de retângulos e quadrados.

Figura 6 - Objeto de Aprendizagem para Área de Retãngulo e Quadrado

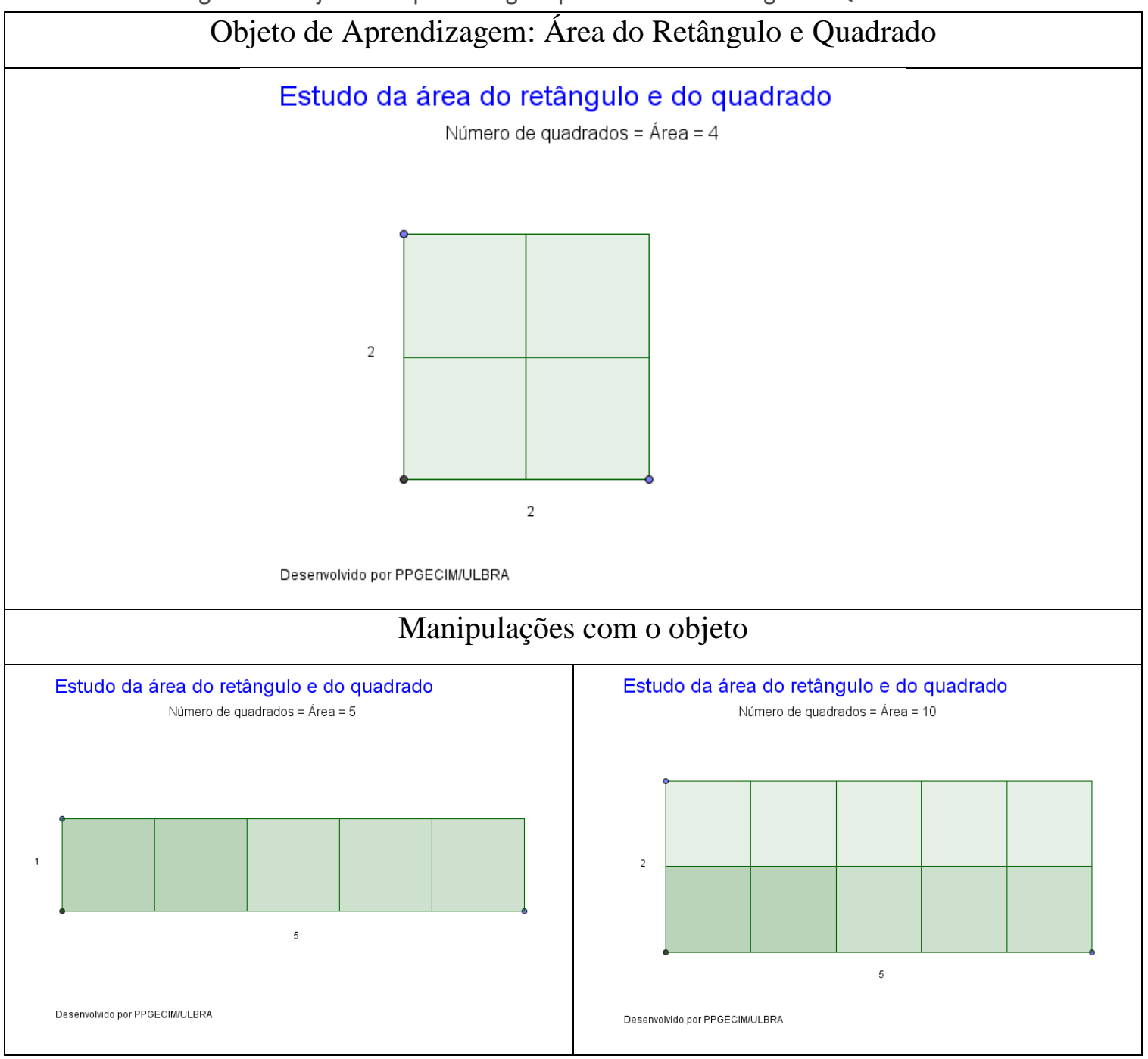

Fonte: Repositório de Objetos de Aprendizagem do PPGECIM. 
O objeto de aprendizagem da Figura 7 apresenta o paralelogramo com a manipulação da sua base e altura e um controle para animação que transforma o objeto em um retângulo, a partir de uma das alturas.

Figura 7 - Objeto de Aprendizagem: Área do Paralelgoramo

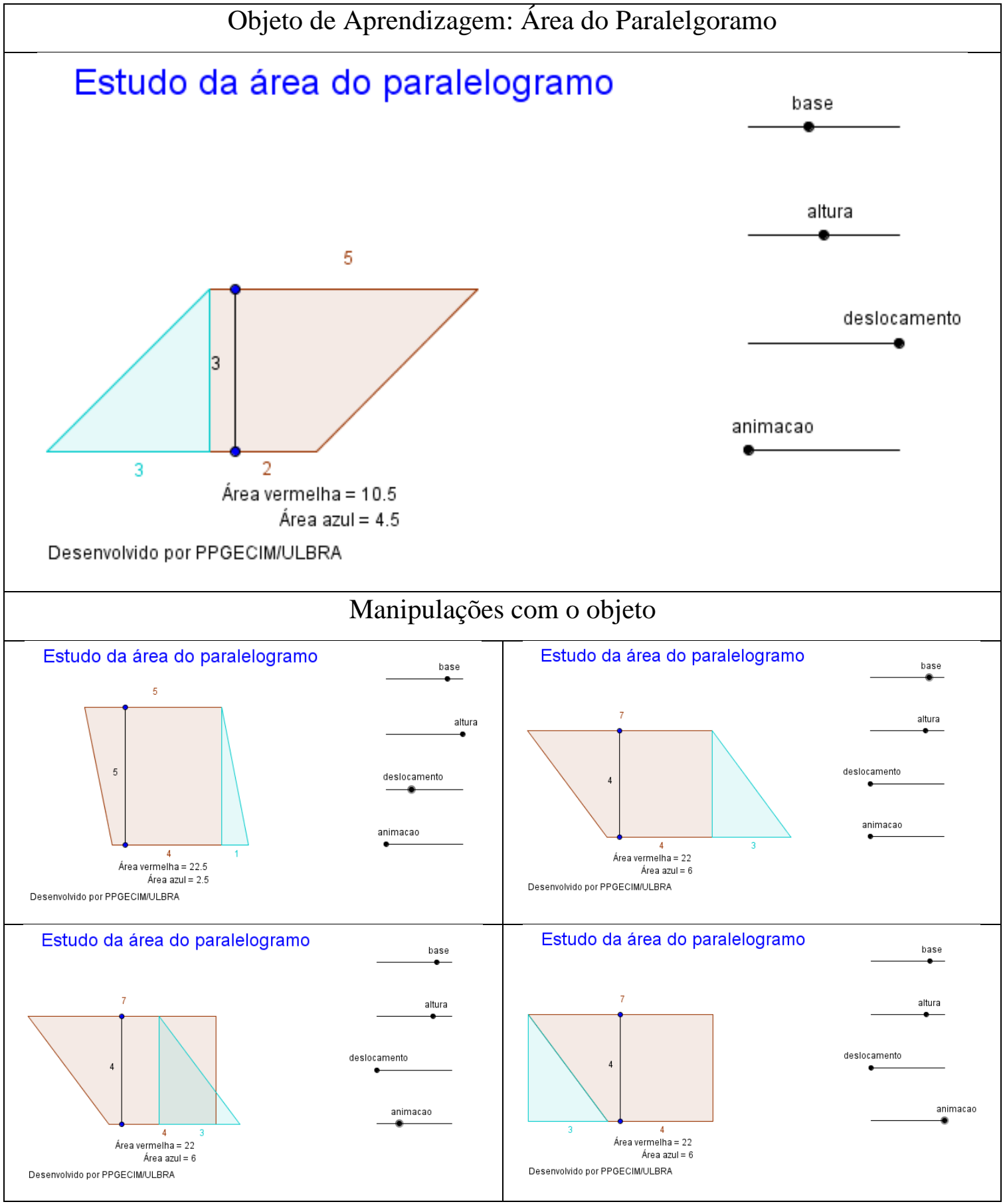

Fonte: Repositório de Objetos de Aprendizagem do PPGECIM. 


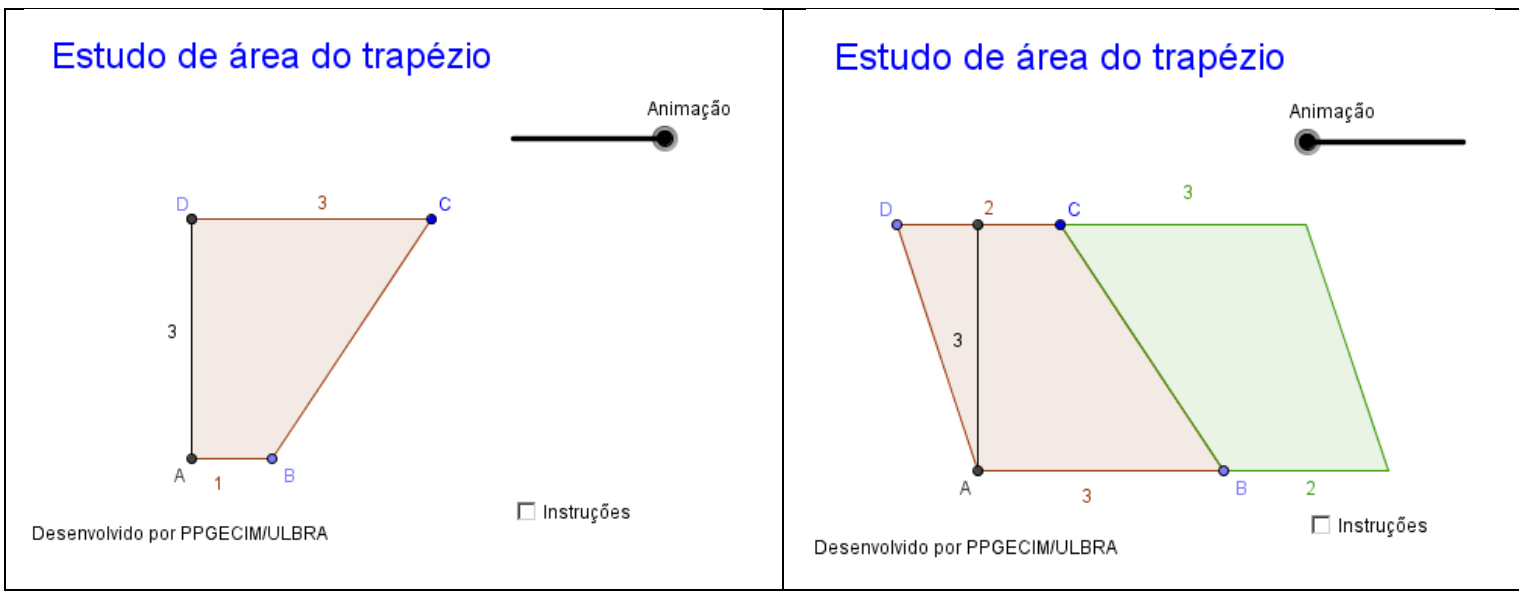

Fonte: Repositório de Objetos de Aprendizagem do PPGECIM.

O objeto de aprendizagem, apresentado na Figura 9, possui três controles, dois para a construção de losangos de diferentes dimensões em relação as diagonais, e um controle de animação que transforma o losango em um retângulo. Objetiva-se que o estudante visualize que a área do losango mede a metade da área do retângulo formado.

Figura 9 - Objeto de Aprendizagem Área do Losango

\section{Objeto de Aprendizagem: Área do Losango}

\section{Estudo da área do losango}
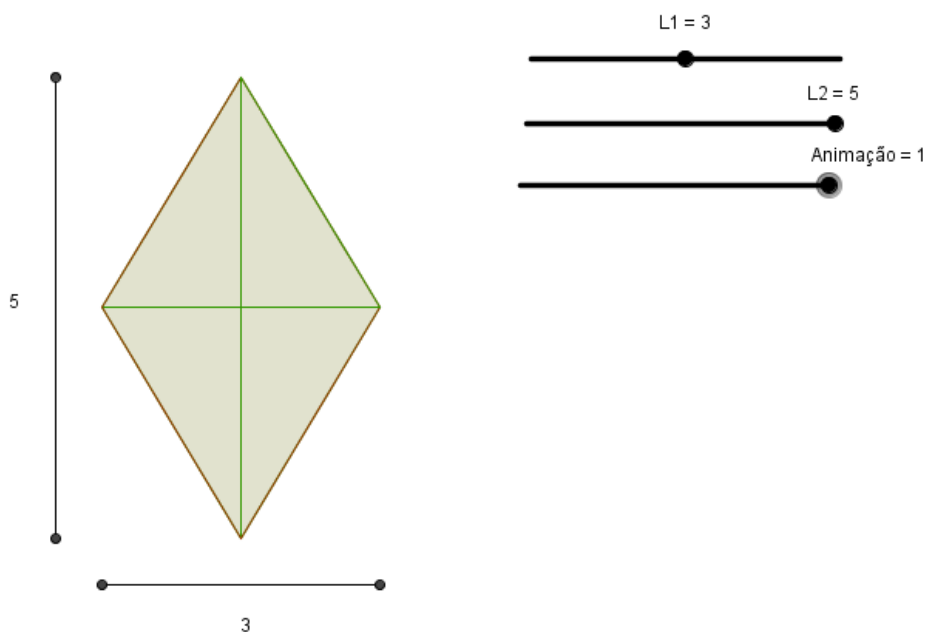

Desenvolvido por PPGECIM/ULBRA 


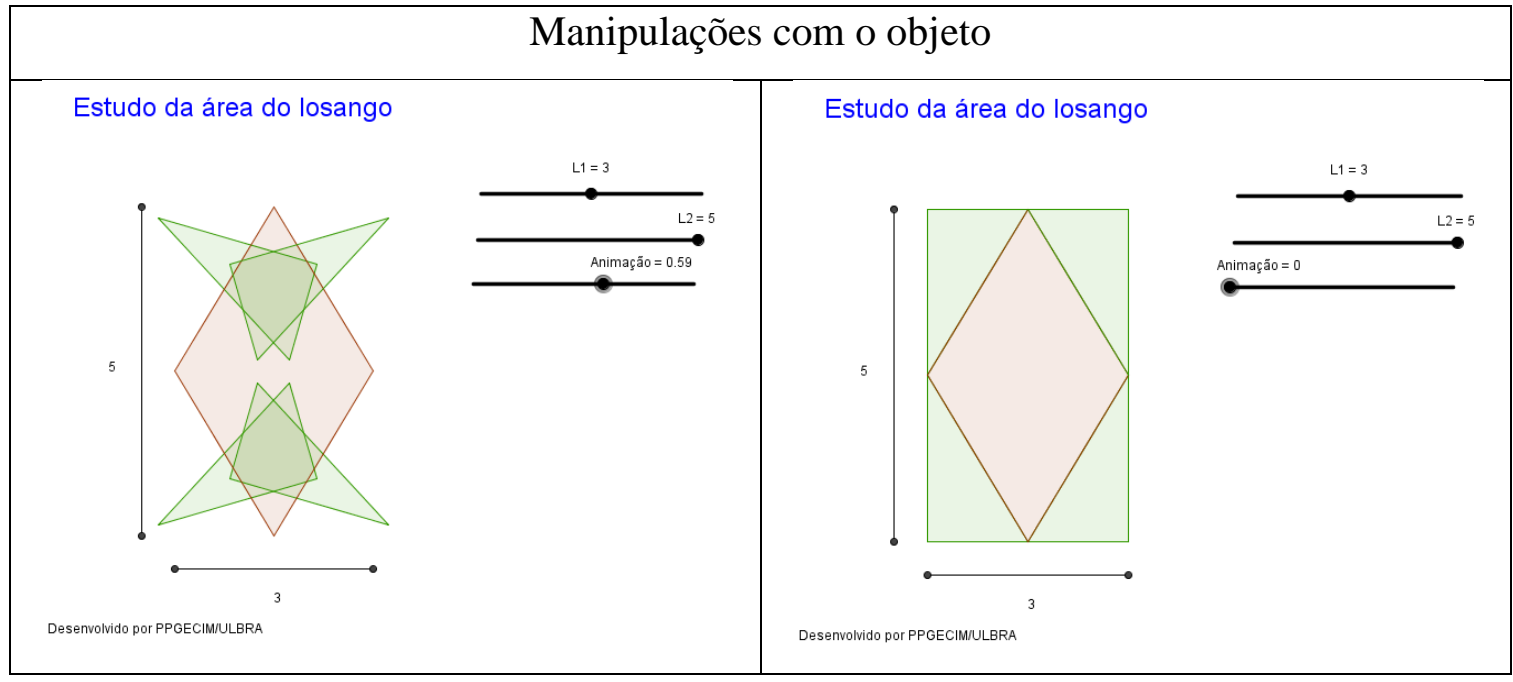

Fonte: Repositório de Objetos de Aprendizagem do PPGECIM.

A Figura 10 apresenta a área de triãngulos. Neste objeto é possível que o estudante visualize a transformação do triângulo em um paralelogramo e que perceba que a medida da área do triãngulo é a metade da área do paralegramo. É possível que o estudante realize as transformações optando por uma das alturas do triângulo em relação a uma das bases. Importante salientar que permite ao estudante observar que dependendo da base escolhida, obtém-se diferentes alturas, permanecendo a mesma medida da área. 
Figura 10 - Objeto de Aprendizagem Área do Triângulo

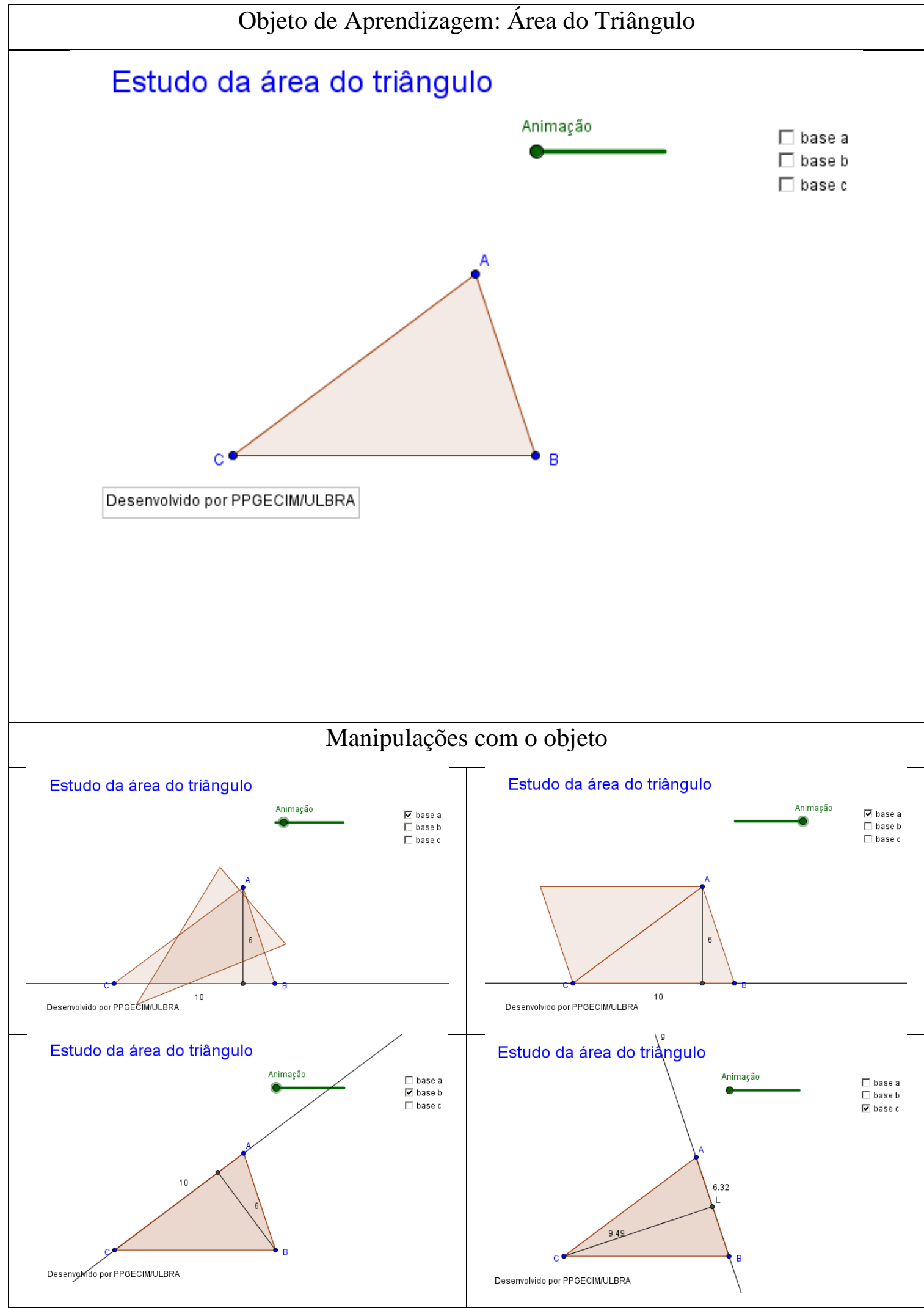

Fonte: Repositório de Objetos de Aprendizagem do PPGECIM. 
A Figura 11 apresenta o objeto de aprendizagem para área do círculo, levando o estudante a visualizar que a medida da área se aproxima da área de um paralelogramo cuja base é a metade do perímetro do círculo e a altura é equivalente ao raio do círculo desenhado. 0 objeto permite, também, que se construa círculos com diferentes medidas de raios, assim como, a decomposição em diferentes números de diâmetros que manipulados formarão o paralelogramo.

Figura 11 - Objeto de Aprendizagem Área do Círculo

Estudo da área do círculo Objeto de Aprendizagem: Área do Círculo




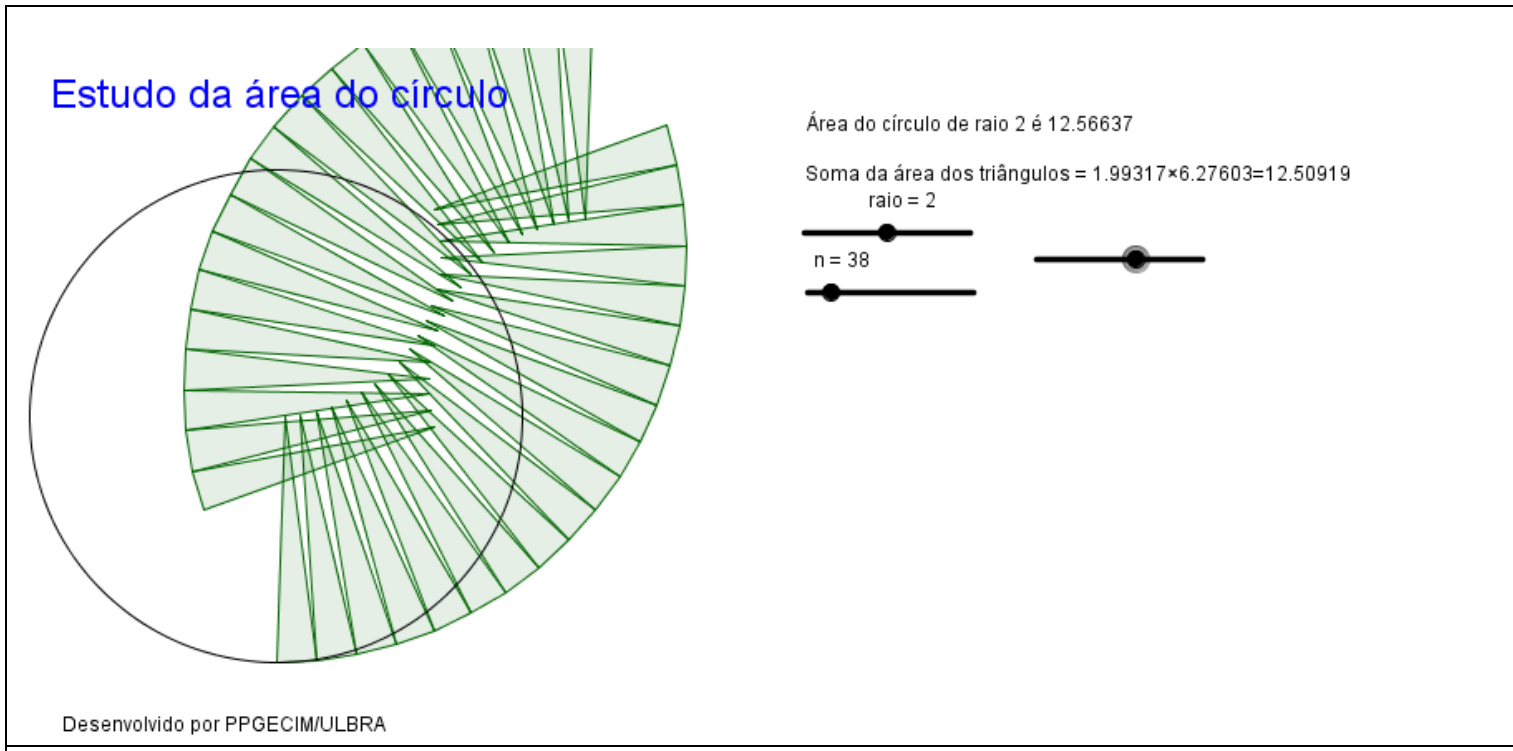

\section{Estudo da área do círculo}

Área do círculo de raio 2 é 12.56637

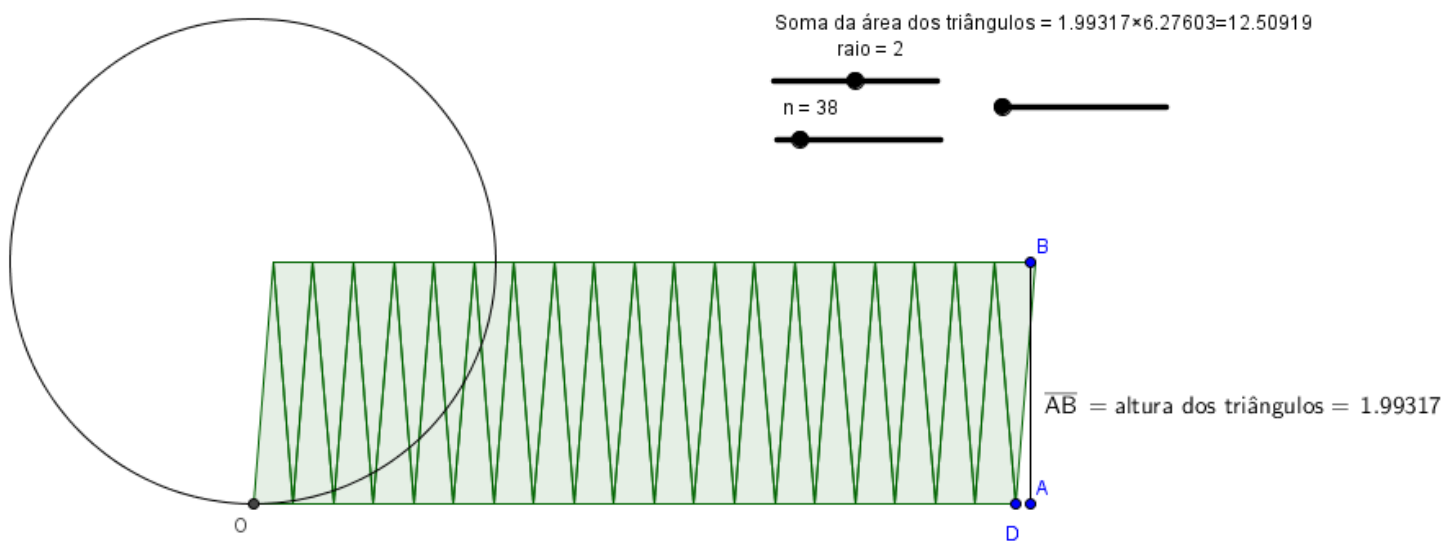

$\overline{\mathrm{OD}}=$ soma das bases dos triângulos $=6.27603=\pi \times 1.99772$

Fonte: Repositório de Objetos de Aprendizagem do PPGECIM.

\section{METODOLOGIA DE INVESTIGAÇÃO}

A sequência didática, composta de 11 objetos de aprendizagem, foi aplicada com estudantes do curso de Licenciatura em Matemática, da Universidade Luterana do Brasil (ULBRA), no primeiro semestre de 2015. Participaram do experimento onze estudantes matriculados na disciplina de Estágio em Matemática I, do quinto semestre do curso (total de oito semestres), com 68 horas aulas, das quais foram utilizados 12 para análise dos objetos de aprendizagem.

Os estudantes manipularam os objetos e foram instigados a tirarem as conclusões que consideravam possíveis com o estudo dos objetos de aprendizagem, trabalhando em três grupos de três estudantes e um grupo de dois. Foi orientado, aos estudantes participantes do experimento, que fosse mantida a sequência didática dos objetos, evitando o acesso aleatório aos mesmos. 
As falas e registros dos estudantes foram registrados através da observação direta dos pesquisadores em sala de aula durante a realização do experimento. Os estudantes estão nomeados como aluno $A$, aluno $B, \ldots$, aluno $L$.

\section{EXPERIMENTO REALIZADO}

O tempo médio de estudo em cada objeto de aprendizagem foi estimado em 10 minutos, porém, no experimento os estudantes despenderam aproximadamente 15 minutos para cada objeto, com os estudantes discutindo, refletindo e tirando conclusões. Salienta-se que o experimento foi realizado com estudantes de licenciatura em Matemática, o que os levou a analisar, também, as possibilidades de utilização com estudantes do Ensino Fundamental.

Os estudantes apresentaram as seguintes dificuldades: no conceito de polígono, na classificação dos quadriláteros, na identificação do modelo matemático do cálculo da área do círculo.

O aluno C declarou: "não consigo concluir o que é um polígono, minha dúvida está em identificar quando é uma linha poligonal fechada e não forma polígono". Observou-se que o aluno apresentava dificuldades quando aparecia a formação de uma linha poligonal fechada com duas áreas internas (Figura 12).

Figura 12 - Linha poligonal fechada

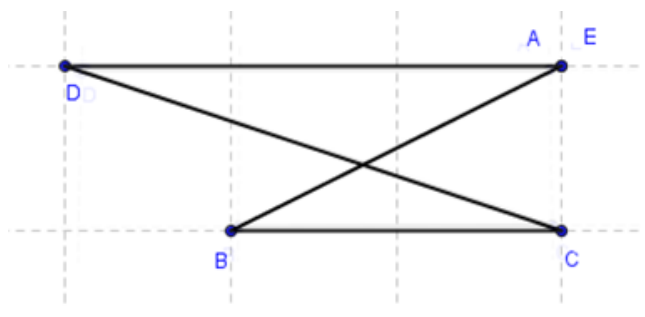

Fonte: a pesquisa.

Outra dificuldade apresentada pelos alunos A, B, D, E, G, H, I, J foi a de identificar quando não era uma linha poligonal porque tinha dois segmentos de reta colineares (Figura 13).

Figura 13 - Linha não poligonal fechada.

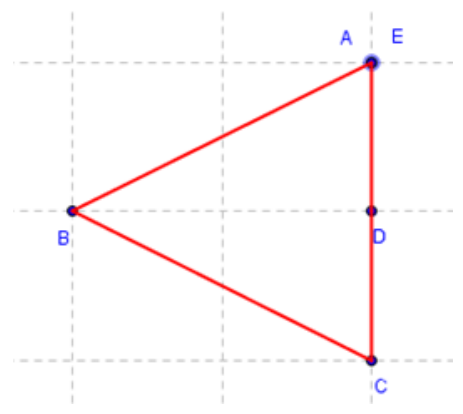

Fonte: a pesquisa.

Os alunos $\mathrm{G}$ e H apresentaram dificuldades na classificação de quadriláteros, pois não conseguiram identificar todas as características dos tipos de 
quadriláteros, levando a uma classificação errada dos mesmos. Dos 11 estudantes, 9 declararam que os paralelogramos podem apresentar ângulos opostos com medidas diferentes, embora o objeto apresentasse os paralelogramos com esta característica (Figura 14).

Figura 14 - Paralelogramo e suas características

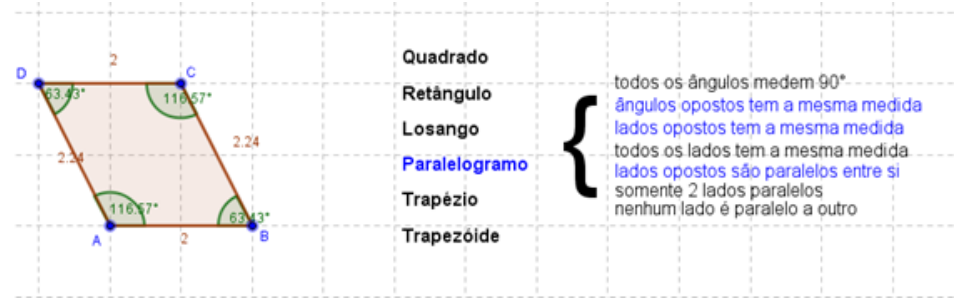

Fonte: a pesquisa.

Os estudantes identificaram as seguintes vantagens do uso dos objetos em relação ao uso da manipulação com lápis e papel: a interatividade permite que sejam testadas várias configurações, sendo possível identificar um padrão e a generalização das características e propriedades matemáticas dos objetos; ao ter objetos prontos aproveita-se melhor o tempo de estudo para análise das características dos mesmos; a visualização leva a conclusão dos modelos matemáticos para a área de figuras planas.

Uma vantagem observada foi no estudo do modelo matemático para o cálculo da área de um triângulo qualquer, com lápis e papel se desenha o triângulo e recorta o mesmo na altura relativa a um dos lados, neste caso o estudante visualiza a fórmula da área da altura em relação a somente um dos lados do triângulo. No objeto em questão é possível visualizar em relação às três alturas do triângulo, permitindo visualizar alturas diferentes que multiplicadas pelos respectivos lados resultam no mesmo valor da área, levando a generalização independente da altura e lado do triângulo.

Quando se utiliza desenhos com lápis e papel para o estudo da fórmula da área do círculo é possível dividir o círculo em um número limitado de partes, já com o objeto de aprendizagem a divisão pode ser feita em duzentas partes (Figura 15), o que possibilita a visualização da fórmula com maior nitidez, o que se pode considerar uma vantagem em relação ao lápis e papel.

Figura 15 - Área do círculo dividido em 200 partes 
Estudo da área do círculo

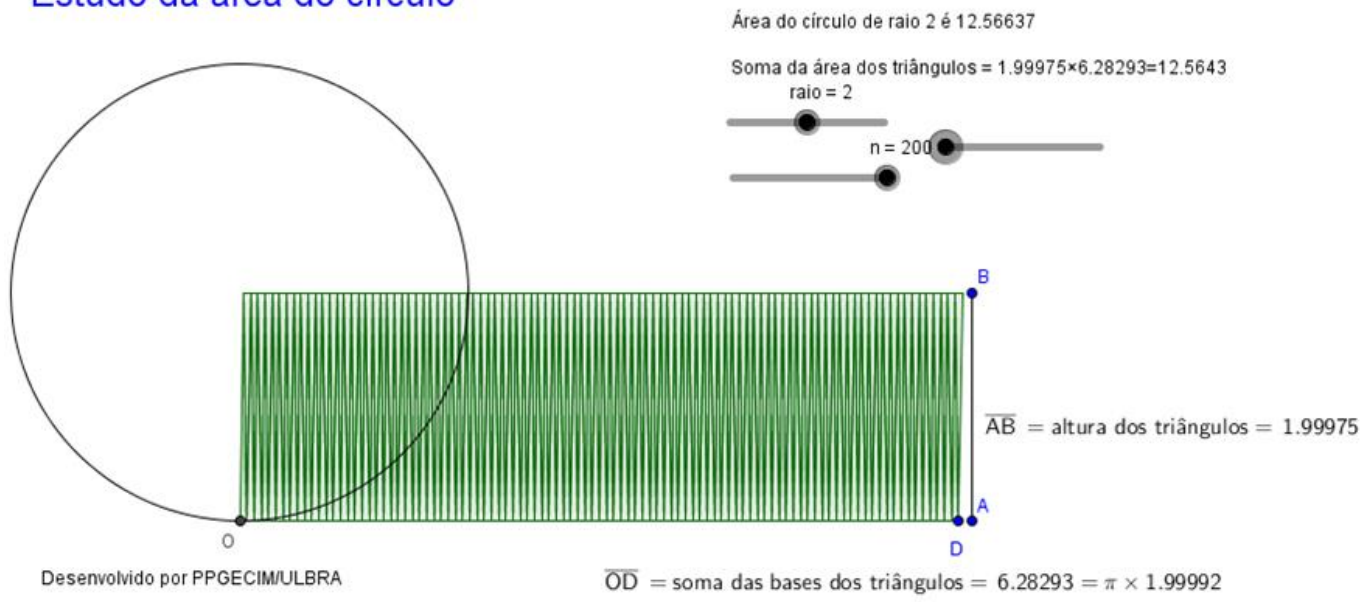

Fonte: a pesquisa.

Todos os estudantes, participantes do experimento, afirmaram que, com certeza, utilizarão estes tipos de objetos de aprendizagem em seus futuros planejamentos de aula, pois consideram que a visualização, interação e comparação entre as várias construções de um mesmo objeto geométrico auxiliam na compreensão dos mesmos.

\section{Conclusão}

O Geogebra é um software adequado à construção de objetos de aprendizagem manipuláveis sem que seja necessário o conhecimento de programação avançada. Importante frisar que os objetos desenvolvidos não devem ser apresentados individualmente, pois os mesmos são baseados no conhecimento da área do paralelogramo e do retângulo, logo, salienta-se a importância da construção de uma sequência didática que apresente os objetos encadeados. Nesse sentido, o desenvolvimento da sequência foi realizada no padrão SCORM, que mostrou-se eficiente, dentro das premissas definidas, atendendo às expectativas de entrega dos conteúdos adequados às necessidades dos alunos.

A escolha do padrão SCORM atendeu a implementação das regras em um padrão aberto de e-learning, o que facilita a difusão das ideias em outras instituições, algo que poderia ser dispendioso e inviável para pequenos projetos de conteúdo utilizando plataformas proprietárias para o e-learning. 


\title{
Area of plane figures with learning objects in geogebra
}

\begin{abstract}
This article describes a didactic sequence developed in digital form, in agreement with SCORM, presented at ILIAS learning management system, to study area of plan figures, using learning objetcts developed at Geogebra software. The aim was make a didactic sequence, with interactive learning objects to college students build the concepts of poligons and the area of plan figures formulas manipulating and observing the objects. The sequence is available at http://matematica.ulbra.br/ilias.
\end{abstract}

KEYWORDS: Geogebra. Digital didactic sequence. Learning objects. Area of plane figure. 


\section{NOTAS}

1 Implementada está sendo utilizada no sentido de desenvolvida, aplicada e avaliada.

2 Granularidade é uma das características dos objetos de aprendizagem, ou seja, o conceito a ser trabalho deve estar completamente contido no objeto de aprendizagem, de modo que possa ser organizado com outros objetos de aprendizagem para conseguir objetivos mais complexos.

\section{REFERÊNCIAS}

ADLNet. SCORM 2004 3rd Edition Content Aggregation model, version 1.0, 2004. Disponível em: <http://www.adlnet.gov/> Acesso em: mai. 2007.

ADLNet. SCORM 2004 3rd Edition Sequencing and Navigation, version 1.0, 2004. Disponível em: <http://www.adlnet.gov/> Acesso em: mai. 2007.

ADLNet. SCORM 2004 3rd Edition Run-Time Environment, version 1.0, 2004. Disponível em: <http://www.adlnet.gov/> Acesso em: mai. 2007.

DOLZ, Joaquim. SCHNEUWLY, Bernard. Gêneros orais e escritos na escola. Campinas/SP: Mercado das Letras, 2004.

GROENWALD, C. L. O.; ZOCH, L.; HOMA, A. I. R.; Sequência Didática com Análise Combinatória no Padrão SCORM. BOLEMA, Rio Claro, v. 22, p. 27 - 56, n. 342009.

HODGINS, H. Wayne. The Future of Learning Objects. Disponível em: <http://www.reusability.org/read/chapters/hodgins.doc>. Acesso em: 17 mai. 2008.

HOHENWARTER, M.; FUCHS, K. Combination of dynamic geometry, algebra and calculus in the software system GeoGebra. Disponível em:

<http://www.geogebra.org/publications/pecs_2004.pdf>. Acesso em: mar. 2014.

HOHENWARTER, M.; PREINER, J. Dynamic Mathematics with GeoGebra. The Journal of Online Mathematics and Its Applications, v. 7, 2007. Disponível em: <http://www.maa.org/external_archive/joma/Volume7/Hohenwarter/index.html >. Acesso em: mar. 2014. 
KAMPFF, A. J. C.; MACHADO, J. C.; CAVEDINI, P. Novas Tecnologias e Educação Matemática. In: X Workshop de Informática na Escola e XXIII Congresso Da Sociedade Brasileira De Computação, 2004, Bahia. Disponível em: <http://www.cinted.ufrgs.br/renote/nov2004/artigos/a12_tecnologias_matemat ica.pdf> Acesso em: 10 jun. 2008.

MORTIMER, L. (Learning) Objects of desire: Promise and practicality. Learning Circuits (April). Disponível em

<http://www.learningcircuits.org/2002/apr2002/mortimer.html>. Acesso em: $15 / 01 / 2011$.

MERRILL, D. Position statement and questions on learning objects research and practice. In: Learning objects technology: Implications for educational research and practice, AERA, April 1-5, New Orleans, LA. Disponível em <http://www.learndev.org/LearningObjectsAERA2002.html>. Acesso em: $15 / 01 / 2012$

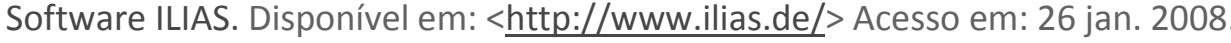

ZABALA, A. A prática educativa: como ensinar. Porto Alegre: Artmed, 1998.

WILEY, D. The instructional use of learning objects. On-line version. Disponível em: <http://reusability.org/read/>. Acesso em: 02/04/2011.

Recebido: 02 de out. de 2014

Aprovado: 03 de mar. de 2016

DOI: $10.3895 /$ rbect.v9n1.2000

Como citar:

HOMA, A. I. R.: GROENWALD, C. L. O. Área de figuras planas com objetos de aprendizagem no Geogebra.

Revista Brasileira de Ensino de Ciência e Tecnologia, v. 9, n. 1, p. 123-147, jan./abr. 2016. Disponível

em: <https://periodicos.utfpr.edu.br/rbect/article/view/2000>. Acesso em: xxx.

Correspondência:

Agostinho laqhan Ryokiti Homa

Rua 12 de outubro, 475, 90680-140, Porto Alegre, Rio Grande do Sul.

Claudia Lisete Oliveira Groenwald

Rua Protásio Alves, 208, 92120, 160, Canoas, Rio Grande do Sul.

Direito autoral: Este artigo está licenciado sob os termos da Licença Creative Commons-Atribuição 4.0 Internacional.

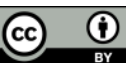

